\title{
A THEOREM ON RIESZ GROUPS
}

\author{
BY \\ J. ROGER TELLER
}

I. Introduction. In this paper, po-group will mean partially ordered torsion free abelian group. A Hahn-type po-group $V$ is defined as follows. Let $\Gamma$ be a po-set, for each $\gamma \in \Gamma$, let $R_{\gamma}$ be a nontrivial po-group. If $v$ is an element of the large direct sum of the $R_{\gamma}$ and $\gamma \in \Gamma, v(\gamma)$ will denote the $\gamma$ th component of $v$. Let $V=V\left(\Gamma, R_{\gamma}\right)$ be the following subset of the large direct sum of the $R_{\gamma}$. An element $v \in V$ if and only if $S(v)=\{\gamma \in \Gamma \mid v(\gamma) \neq 0\}$ contains no infinite ascending sequences; that is, every nonempty subset of $S(v)$ contains maximal elements. Since the union of two subsets of $\Gamma$ each satisfying the maximum condition, also satisfies the maximum condition, it follows that $V$ is a subgroup of the large direct sum of the $\boldsymbol{R}_{\boldsymbol{r}}$.

For each $v \in V$, let

$$
M(v)=\{\gamma \in \Gamma \mid v(\gamma) \neq 0 \text { and } v(\alpha)=0 \text { for all } \alpha>\gamma\} .
$$

If $\gamma \in M(v)$, then $v(\gamma)$ is called a maximal component of $v$. A nonzero element $v \in V$ is defined to be positive if each maximal component $v(\gamma)$ of $v$ is positive with respect to the partial order of $R_{\gamma}$.

P. Conrad, J. Harvey, and C. Holland [1, p. 145] have shown that $V$, ordered as above, is a po-group. They have also shown that $V$ is a lattice ordered group if each $R_{\gamma}$ is a totally ordered group (o-group) and $\Gamma$ is a root system, i.e., for each $\gamma \in \Gamma,\{\alpha \in \Gamma \mid \alpha>\gamma\}$ is a chain.

In [2], the author considered special cases of Hahn-type po-groups as examples of Riesz groups. A Riesz group $G$ is defined as a semiclosed po-group ( $g \in G$ and $n g \geqq 0$ for $n>0$ implies $g \geqq 0$ ) that satisfies the Riesz interpolation property. That is, whenever $x_{1}, \ldots, x_{m}, y_{1}, \ldots, y_{n}$ are elements of $G$ and $x_{i} \leqq y_{j}, i=1, \ldots, m$; $j=1, \ldots, n$, then there is an element $z \in G$ such that $x_{i} \leqq z \leqq y_{j}$. We list in [2] some conditions that are equivalent to the Riesz interpolation property. The condition we find most convenient to use is the decomposition property: if $x, u, v \in G$ and $0 \leqq x \leqq u+v$ where $u \geqq 0, v \geqq 0$, then there exists elements $\bar{u}, \bar{v} \in G$ such that $x=\bar{u}+\bar{v}$ and $0 \leqq \bar{u} \leqq u, 0 \leqq \bar{v} \leqq v$.

The purpose of this paper is to determine necessary and sufficient conditions so the Hahn-type po-group $V\left(\Gamma, R_{y}\right)$ will be a Riesz group. In doing so we answer in the affirmative the following question which we posed at the end of [2]. If $\Gamma$ is an arbitrary po-set and for each $\gamma \in \Gamma, R_{\gamma}$ is the naturally ordered group of real numbers, is $V\left(\Gamma, R_{\gamma}\right)$ a Riesz group?

Presented to the Society, September 2, 1966; received by the editors March 31, 1966. 
Since every lattice ordered group is a Riesz group, the conditions of Theorem $2.2[1$, p. 145] are sufficient for $V$ to be a Riesz group. Our results reflect this and are a generalization of this theorem to Riesz groups.

II. The main theorem. In this section we establish the terminology and notations to be used and state the main theorem and some immediate corollaries.

Let $\Gamma$ be a po-set. If $\gamma$ and $\delta$ are elements of $\Gamma$, then $\gamma$ and $\delta$ are disjoint if $\gamma \delta \delta$ and $\delta \leqslant \gamma$. If $\gamma$ and $\delta$ are disjoint we write $\gamma \| \delta$. A nonempty subset $A$ of $\Gamma$ is a disjoint set if $\gamma \| \delta$ for every pair of distinct elements $\gamma, \delta \in A$. If $\beta \in \Gamma$ and $(\gamma, \delta) \in \Gamma \times \Gamma$, the notation $\beta<(\gamma, \delta)$ will mean $\beta<\gamma$ and $\beta<\delta$.

An o-group $G$ is order dense, or merely dense, if $x<y$ in $G$ implies there is an element $z \in G$ such that $x<z<y$. An o-group $G$ that is not dense will be called discrete. If $G$ is a trivially ordered group, then $G$ will be called trivial.

For each $\gamma \in \Gamma$, let $R_{\gamma}$ be a trivially ordered group or an o-group. The element $\gamma$ will be called a dense (discrete, trivial) component if $R_{\gamma}$ is dense (discrete, trivial). A nontrivial component will often be called ordered.

Let $\Delta \subseteq \Gamma \times \Gamma$ and $\pi_{i}, i=1,2$, denote the projections. Then $\Delta$ is totally disjoint if

(i) $\pi_{1} \Delta \cap \pi_{2} \Delta=\varnothing$,

(ii) $\pi_{1} \Delta \cup \pi_{2} \Delta$ is a disjoint set, and

(iii) $\pi_{1} \Delta \cup \pi_{2} \Delta$ contains no trivial components.

If $A$ and $B$ are nonempty subsets of $\Gamma$, then $B$ covers $A$ if, for each $\alpha \in A$, there is $\beta \in B$ such that $\beta \geqq \alpha$. A totally disjoint set $\Delta$ is complete if, whenever $A$ is a disjoint subset of $\{\alpha \in \Gamma \mid \alpha<(\gamma, \delta)$ for some $(\gamma, \delta) \in \Delta\}$ and $A$ contains only trivial components, there is a disjoint subset

$$
B \subseteq\left\{\alpha \in \Gamma \mid \alpha<(\gamma, \delta) \text { for some }(\gamma, \delta) \in \pi_{1} \Delta \times \pi_{2} \Delta\right\}
$$

such that no element of $B$ is trivial and $B$ covers $A$. A subset $S$ of $\Gamma$ is complete if, whenever $A$ is a disjoint subset of $\{\alpha \in \Gamma \mid \alpha<\beta$ for some $\beta \in S\}$ and $A$ contains only trivial components, there is a disjoint subset $B \subseteq\{\alpha \in \Gamma \mid \alpha<\beta$ for some $\beta \in S\}$ such that no element of $B$ is trivial and $B$ covers $A$. Finally, if $T$ is a nonempty subset of $\Gamma$ then we define $T^{*}=\{\alpha \in \Gamma \mid \alpha \leqq \gamma$ for some $\gamma \in T\}$. The notation $\alpha<T$ means $\alpha<\gamma$ for all $\gamma \in T$.

THEOREM. Let $\Gamma$ be a po-set and for each $\gamma \in \Gamma$, let $R_{\gamma}$ be a trivially ordered group or an o-group. Then $V=V\left(\Gamma, R_{\gamma}\right)$ is a Riesz group if and only if every totally disjoint subset of $\Gamma \times \Gamma$ and every disjoint set of discrete components of $\Gamma$ is complete.

We first note that, since every o-group is semiclosed and $V$ is a subgroup of the large direct sum of the $R_{\gamma}$, the po-group $V$ is semiclosed. Thus, in all that follows we concern ourselves with the decomposition property.

Our first corollary answers the question at the end of [2].

COROLlARY 1. If $\Gamma$ is an arbitrary po-set and for each $\gamma \in \Gamma, R_{\gamma}$ is an o-group then $V\left(\Gamma, R_{\gamma}\right)$ is a Riesz group. 
Proof. The conditions are clearly satisfied since there are no trivial components.

If $\Gamma$ is taken to be a root system, then Corollary 1 shows our results are consistent with those of Theorem 2.2 [1, p. 145]. The next corollary generalizes this.

COROLLARY 2. If $\Gamma$ is a root system and for each $\gamma \in \Gamma, R_{\gamma}$ is a dense o-group or a trivially ordered group, then $V\left(\Gamma, R_{\gamma}\right)$ is a Riesz group.

Proof. In the root system $\Gamma, \alpha<(\gamma, \delta)$ cannot occur if $\gamma \| \delta$. Thus, every totally disjoint subset $\Gamma \times \Gamma$ is complete.

The conditions of the theorem are somewhat complicated so we offer the following examples to show what the conditions do.

EXAMPLE 1. Let $\Gamma=\{\alpha, \beta, \gamma\}$ where $\beta>\alpha, \gamma>\alpha$ and $\beta \| \gamma$ (Figure 1).

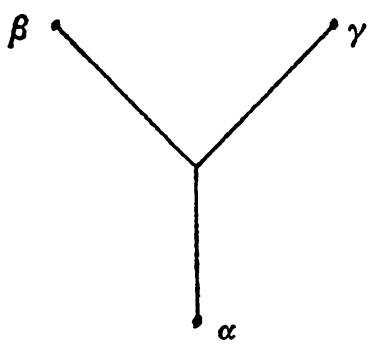

FiguRE 1

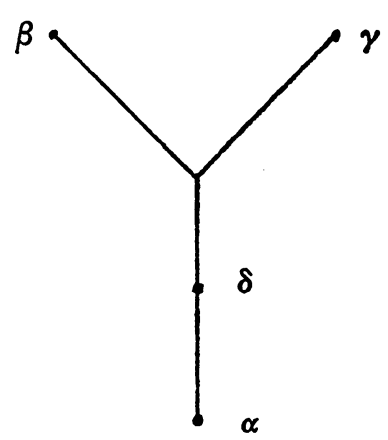

Figure 2

Let $R_{\beta}=R_{\gamma}$ be the naturally ordered real numbers and $R_{\alpha}$ the trivially ordered real numbers. Then $V=R_{\alpha} \oplus R_{\beta} \oplus R_{\gamma}$ (the direct sum) is a po-group but not a Riesz group. This can be seen by letting $x=(1,0,1), u=(0,0,1)$ and $v=(0,1,0)$. Then $0<x<u+v$ where these elements are strictly positive. If $x=\bar{u}+\bar{v}$ where $\bar{u} \in V, \bar{v} \in V$ and $0 \leqq \bar{u} \leqq u, 0 \leqq \bar{v} \leqq v$, then $\bar{u}(\beta)=\bar{v}(\beta)=0, \bar{v}(\gamma)=0$ and $\bar{u}(\gamma)=1$. Thus, $\bar{v}(\alpha)=0$ and $\bar{u}(\alpha)=1$ so that $\bar{v}=0$ and $\bar{u}=x$. However, $x \leq u$ so the decomposition property does not hold. This is because the totally disjoint set $\Delta=\{(\beta, \gamma)\}$ is not complete. That is, there is no way to cover $A=\{\alpha\}$ by a subset of $\pi_{1} \Delta \times \pi_{2} \Delta$.

We can correct this by introducing another element $\delta$ where $\alpha<\delta<(\beta, \gamma)$ (Figure 2) and by taking $R_{\delta}$ to be the naturally ordered reals. Then $\Delta=\{(\beta, \gamma)\}$ is complete since $B=\{\delta\}$ covers $A=\{\alpha\}$ and $\delta$ is ordered and $V=R_{\alpha} \oplus R_{\delta} \oplus R_{\beta} \oplus R_{\gamma}$ is a Riesz group.

Thus, we see that for each trivial $\alpha<(\beta, \gamma)$ where $\beta \| \gamma$ and both are ordered, there must exist an ordered $\delta$ such that $\alpha<\delta<(\beta, \gamma)$. However, the next example shows that this condition, by itself, is not sufficient.

Example 2. Let $\Gamma=\left\{\beta, \gamma, \delta_{i}, \alpha_{i}\right\}$ where $i=1,2,3, \ldots$ Partially order $\Gamma$ as, $\beta \| \gamma, \delta_{i}<(\beta, \gamma), \alpha_{i}<(\beta, \gamma)$ for all $i, \alpha_{i} \| \alpha_{j}$ if $i \neq j, \delta_{i}<\delta_{j}$ if $i<j, \alpha_{i}<\delta_{j}$ if $i \leqq j$ (Figure 3). For each $i$, let $R_{\alpha_{i}}$ be the trivially ordered real numbers and for $\lambda \in \Gamma, \lambda \neq \alpha_{i}$ let $R_{\lambda}$ 


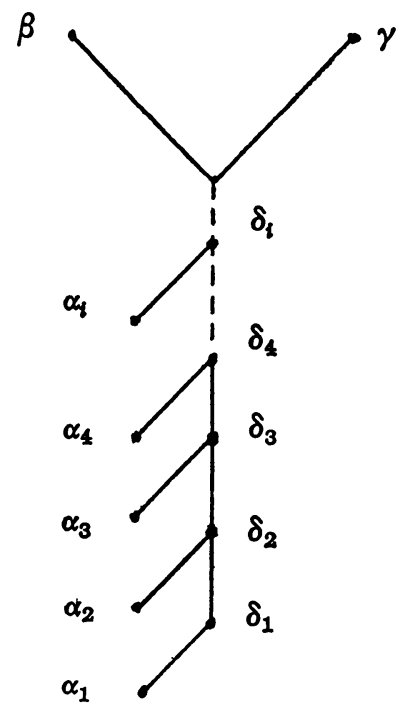

FIGURE 3

be the naturally ordered reals. Then $V=V\left(\Gamma, R_{\gamma}\right)$ is not a Riesz group. For let $x, u, v$ be defined as

$$
\begin{aligned}
& x(\lambda)=1 \text { if } \lambda=\gamma, \\
& u(\lambda)=1 \quad \text { if } \lambda=\gamma, \\
& =1 \text { if } \lambda=\alpha_{i}, i=1,2, \ldots \text {, } \\
& =0 \text { otherwise; } \\
& =0 \text { otherwise; } \\
& v(\lambda)=1 \quad \text { if } \lambda=\beta . \\
& =0 \text { otherwise. }
\end{aligned}
$$

Then $0<x<u+v$. However, every definition of $\bar{u}$ and $\bar{v}$ that satisfies $x=\bar{u}+\bar{v}$ and $0 \leqq \bar{u} \leqq u, 0 \leqq \bar{v} \leqq v$, must be such that either the supports $S(\bar{u})$ or $S(\bar{v})$ contain an infinite ascending sequence. Thus, there are no elements of $V$ that satisfy the decomposition property for $x$. This is because $A=\left\{\alpha_{i} \mid i=1,2, \ldots\right\}$ is a disjoint subset of trivial components of $\{\lambda \in \Gamma \mid \lambda<(\beta, \gamma)\}$ where, clearly, $\{(\beta, \gamma)\}$ is totally disjoint. Now, $A$ can be covered by $\left\{\delta_{i} \mid i=1,2, \ldots\right\}$ but no disjoint subset of this latter set covers $A$. Thus, $\{(\beta, \gamma)\}$ is not complete.

The above examples involved dense o-groups. We now give a very simple example to show why every disjoint set of discrete components must be complete.

EXAMPLE 3. Let $\Gamma=\{\alpha, \beta\}$ with $\alpha<\beta, R_{\beta}$ the naturally ordered integers and $R_{\alpha}$ the trivially ordered reals.

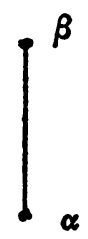


Thus, $V=R_{\alpha} \oplus R_{\beta}$ is not a Riesz group. For let $x=(1,1), u=v=(0,1)$. Then $0<x<u+v$. If $x=\bar{u}+\bar{v}$ where $0 \leqq \bar{u} \leqq u, 0 \leqq \bar{v} \leqq v$ then, without loss of generality, $\bar{u}(\beta)=1$ so $\bar{v}(\beta)=0, \bar{v}(\alpha)=0$ and $\bar{u}(\alpha)=1$. Thus, $\bar{u}=(1,1)$

In an attempt to simplify the constructions to follow we first prove the following

LEMMA. Let $\Gamma$ be a po-set and for each $\gamma \in \Gamma$, let $R_{\gamma}$ be a trivially ordered group or a dense o-group. Then $V=V\left(\Gamma, R_{\gamma}\right)$ is a Riesz group if and only if every totally disjoint subset of $\Gamma \times \Gamma$ is complete.

III. Proof of lemma-sufficiency. For each $\gamma \in \Gamma$, let $R_{y}$ be a dense o-group or a trivially ordered group and suppose every totally disjoint subset of $\Gamma \times \Gamma$ is complete. Let $x, u$ and $v$ be positive elements of $V=V\left(\Gamma, R_{y}\right)$ such that $0 \leqq x \leqq u+v$. If $x=0$ or $x=u+v$, there is nothing to prove so it may be assumed that $0<x<u+v$ and $0<u, 0<v$. Let $w=u+v-x$. Thus, $M(x), M(u), M(v), M(w)$ are nonempty collections of the dense components.

In the following, we repeatedly use the fact that if $\gamma \in M(x) \cup M(w)$, then there exists $\beta \in M(u) \cup M(v)$ such that $\beta \geqq \gamma$.

We first decompose $\Gamma$ into three disjoint subsets. Let

$$
\Gamma_{0}=M(x)^{*} \cap M(u)^{*} \cap M(v)^{*} \cap M(w)^{*}
$$

and $\Gamma_{1}=\Gamma \mid \Gamma_{0}$. Let $\Gamma_{2}=\left\{\alpha \in \Gamma_{0} \mid C \cap M(x)=\varnothing\right.$ or $C \cap M(w)=\varnothing$ for all maximal chains $C \subseteq \Gamma$ such that $\alpha \in C\}$ and $\Gamma_{3}=\Gamma_{0} \mid \Gamma_{2}$.

We now construct $\bar{u}, \bar{v} \in V$ such that $\bar{u}+\bar{v}=x, 0 \leqq \bar{u} \leqq u$ and $0 \leqq \bar{v} \leqq v$ by defining $\bar{u}(\alpha), \bar{v}(\alpha)$ for each $\alpha \in \Gamma$.

Thus, let $\alpha \in \Gamma$. If $\alpha \in \Gamma_{1}$ and

(1) $\alpha \notin M(x)^{*}$, let $\bar{u}(\alpha)=\bar{v}(\alpha)=0$,

(2) $\alpha \in M(x)^{*} \mid M(w)^{*}$, let $\bar{u}(\alpha)=u(\alpha), \bar{v}(\alpha)=v(\alpha)$,

(3) $\alpha \in M(x)^{*} \cap M(w)^{*}$ but

(i) $\alpha \notin M(u)^{*}$, let $\bar{u}(\alpha)=0, \bar{v}(\alpha)=x(\alpha)$,

(ii) $\alpha \notin M(v)^{*}$, let $\bar{u}(\alpha)=x(\alpha), \bar{v}(\alpha)=0$.

It frequently happens, as in (i) and (ii) above, that two definitions are duals of each other. We will denote this by merely saying, "dual".

Now define $\Delta \subseteq M(x) \times M(w)$ as, $(\gamma, \delta) \in \Delta$ if and only if $\gamma \| \delta$ and there is an element $\lambda \in \Gamma_{2}$ such that $\lambda<(\gamma, \delta)$. Let $J$ denote the set of maximal elements of $\Gamma_{2} \cap[S(x) \cup S(u) \cup S(v)]$ and $A$ the trivial components of $J$.

Let $A(u)=\{\alpha \in A \mid$ there is $(\gamma, \delta) \in \Delta$ such that $\alpha<(\gamma, \delta), \gamma \in M(u)$ and $\delta \in M(v)\}$. Let $A(v)=\{\alpha \in A \backslash A(u) \mid$ there is $(\gamma, \delta) \in \Delta$ such that $\alpha<(\gamma, \delta), \gamma \in M(v)$ and $\delta \in M(u)\}$. Define $\Delta(u)$ as $(\gamma, \delta) \in \Delta(u)$ if and only if $(\gamma, \delta) \in \Delta, \gamma \in M(u), \delta \in M(v)$ and there is $\alpha \in A(u)$ such that $\alpha<(\gamma, \delta)$. Then $\Delta(u)$ is totally disjoint, $A(u)$ is a disjoint subset of trivial components of $\{\lambda \in \Gamma \mid \lambda<(\gamma, \delta) \in \Delta(u)\}, \pi_{1} \Delta(u) \subseteq M(x)$ $\cap M(u)$ and $\pi_{2} \Delta(u) \subseteq M(w) \cap M(v)$. Thus, there is a disjoint subset $B_{1}$ of ordered 
components of $\left\{\lambda \in \Gamma \mid \lambda<(\gamma, \delta) \in \pi_{1} \Delta(u) \times \pi_{2} \Delta(u)\right\}$ such that $B_{1}$ covers $A(u)$. Clearly, $B_{1} \subseteq \Gamma_{0}$ and, without loss of generality, we may assume for each $\beta \in B_{1}$ there is $\alpha \in A(u)$ such that $\beta>\alpha$. Thus, $B_{1} \subseteq \Gamma_{2}$.

By a dual argument, $\Delta(v)$ exists and is totally disjoint. Moreover, $A(v)$ is a disjoint subset of trivial components of $\left\{\lambda \in \Gamma_{\lambda}<(\gamma, \delta) \in \Delta(v)\right\}$. Thus, there is a disjoint subset $B_{2}$ of ordered components of $\left\{\lambda \in \Gamma \mid \lambda<(\gamma, \delta) \in \pi_{1} \Delta(v) \times \pi_{2} \Delta(v)\right\}$ such that $B_{2}$ covers $A(v)$. As above, $B_{2} \subseteq \Gamma_{2}$.

Let $B_{3}=\{\eta \in M(u) \cup M(v) \mid$ there is $\alpha \in A \backslash[A(u) \cup A(v)]$ and $(\gamma, \delta) \in \Delta$ such that $\alpha<\eta<(\gamma, \delta)\}$. Then $B_{3}$ is a disjoint subset of ordered components and $B_{3} \subseteq \Gamma_{2}$. Let $A_{2}=\left\{\alpha \in A \backslash[A(u) \cup A(v)] \mid \alpha<\beta\right.$, for some $\left.\beta \in B_{3}\right\}$.

Now, if $\alpha \in A_{3}=A \backslash\left[A(u) \cup A(v) \cup A_{2}\right]$, then $\gamma \in M(u)$ and $\delta \in M(u)$ for all $(\gamma, \delta) \in \Delta$ such that $\alpha<(\gamma, \delta)$, or $\gamma \in M(v)$ and $\delta \in M(v)$ for all $(\gamma, \delta) \in \Delta$ such that $\alpha<(\gamma, \delta)$. Moreover, there exists an element $\eta \in M(u) \cup M(v)$ such that $\alpha<\eta$ and $\eta<\gamma \in M(x)$ or $\eta<\delta \in M(w)$, but not both. Let $A(x)=\left\{\alpha \in A_{3} \mid\right.$ there exist elements $\eta \in M(u) \cup M(v)$ and $\gamma \in \pi_{1} \Delta$ such that $\left.\alpha<\eta<\gamma\right\}$ and $A(w)=A_{3} \mid A(x)$.

We construct a new set $\Delta(x)$ as follows. For each $\alpha \in A(x)$ pick $(\beta, \delta) \in \Delta$ such that $\alpha<(\beta, \delta)$, and pick $\eta \in M(u) \cup M(v)$ such that $\alpha<\eta<\gamma$ for some $\gamma \in \pi_{1} \Delta$. Let $(\eta, \delta) \in \Delta(x)$. Then $\Delta(x)$ is totally disjoint and $A(x)$ is a disjoint subset of trivial components of $\{\lambda \in \Gamma \mid \lambda<(\gamma, \delta) \in \Delta(x)\}$. Thus, there is a disjoint subset $B_{4}$ of ordered components of $\left\{\lambda \in \Gamma \mid \lambda<(\gamma, \delta) \in \pi_{1} \Delta \times \pi_{2} \Delta\right\}$ such that $B_{4}$ covers $A(x)$. Clearly, $B_{4} \subseteq \Gamma_{0}$ and again we assume for each $\beta \in B_{4}$ there is an element $\alpha \in A(x)$ such that $\beta>\alpha$. Thus, $B_{4} \subseteq \Gamma_{2}$.

Finally, if $\alpha \in A(w)$ and $\alpha<\eta \in M(u) \cup M(v)$, then $\eta<\delta \in \pi_{2} \Delta$. Construct $\Delta(w)$ similar to $\Delta(x)$ above. Then there exists a disjoint set $B_{5}$ of ordered components such that $B_{5}$ covers $A(w)$ and $B_{5} \subseteq \Gamma_{2}$. If any of $A(u), A(v), A(x)$ or $A(w)$ is empty, we take the corresponding $B_{i}$ to be the empty set.

Thus, $A$ is covered in $\Gamma_{2}$ by $\bigcup B_{i}, i=1, \ldots, 5$ where each $B_{i}$ is disjoint. Let $B^{\prime}$ be the maximal components of $\left(\cup B_{i}\right) \cup(J \backslash A)$. Then $B^{\prime} \subseteq \Gamma_{2}$.

(4) If $\alpha \in \Gamma_{2} \mid\left(B^{\prime}\right)^{*}$, let $\bar{u}(\alpha)=\bar{v}(\alpha)=0$.

(5) (i) If $\alpha \in B^{\prime} \cap M(u)$, let $\bar{u}(\alpha)=0, \bar{v}(\alpha)=x(\alpha)$,

(ii) dual of (i).

(6) (i) If $\alpha \in B^{\prime} \backslash[M(u) \cup M(v)]$ and for all $(\gamma, \delta) \in \Delta$ such that $\alpha<(\gamma, \delta)$, no $\eta \in M(v)$ exists such that $\alpha<\eta \leqq \gamma$, let $\bar{u}(\alpha)<\{0, u(\alpha), x(\alpha)\}$ and $\bar{v}(\alpha)=x(\alpha)-\bar{u}(\alpha)$,

(ii) dual of (i).

(7) If $\alpha \in B^{\prime} \backslash[M(u) \cup M(v)], \beta \in M(u)$ such that $\alpha<\beta \leqq \gamma_{1}$ for some $\left(\gamma_{1}, \delta_{1}\right) \in \Delta$, $\eta \in M(v)$ such that $\alpha<\eta \leqq \gamma_{2}$ for some $\left(\gamma_{2}, \delta_{2}\right) \in \Delta$ where $\alpha<\left(\gamma_{i}, \delta_{i}\right), i=1,2$ and

(i) for all $(\gamma, \delta) \in \Delta$ such that $\alpha<(\gamma, \delta)$, no $\beta \in M(u)$ is such that $\alpha<\beta \leqq \delta$, let $\bar{u}(\alpha)<\{0, u(\alpha), x(\alpha)\}$ and $\bar{v}(\alpha)=x(\alpha)-\bar{u}(\alpha)$,

(ii) dual of (i).

(8) If $\alpha \in \Gamma_{2}, \alpha<\eta \in B^{\prime}$ and $\beta \in M(u)$ for all $\beta \in B^{\prime}$ such that $\beta>\alpha$, let $\bar{u}(\alpha)=0$, $\bar{v}(\alpha)=x(\alpha)$.

(9) For all other $\alpha \in \Gamma_{2}$, let $\vec{u}(\alpha)=x(\alpha), \bar{v}(\alpha)=0$. 
Let $K=\left\{\lambda \in \Gamma_{3} \mid \lambda \in M(x) \cup M(w) \cup M(u) \cup M(v)\right\}, K^{\prime}=\left\{\sigma \in \Gamma_{3} \mid \sigma<\lambda\right.$ for some $\lambda \in K\}$ and $L=\Gamma_{3} \mid K^{*}$. Let $\alpha \in K$.

(10) (i) If $\alpha \in M(x)$ and $\alpha \notin M(u) \cup M(v) \cup M(w)$, let $\bar{u}(\alpha)=x(\alpha), \bar{v}(\alpha)=0$.

(ii) If $\alpha \in M(x) \cap M(v)$ and $\alpha \notin M(u) \cup M(w)$, let $\bar{u}(\alpha)=x(\alpha), \bar{v}(\alpha)=0$,

(iii) dual of (ii).

(11) (i) If $\alpha \in M(v)$ and $\alpha \notin M(u) \cup M(x) \cup M(w)$, let $\bar{u}(\alpha)=x(\alpha), \bar{v}(\alpha)=0$,

(ii) dual of (i).

(12) If $\alpha \in M(w)$, then $x(\alpha)<u(\alpha)+v(\alpha)$. If $b \in R_{\alpha}$ such that

$$
b<\{0, x(\alpha), u(\alpha), v(\alpha)\}
$$

then by applying the decomposition property to $0<x(\alpha)-2 b<(u(\alpha)-b)+(v(\alpha)-b)$, there are elements $y, z \in R_{\alpha}$ such that $x(\alpha)-2 b=y+z$ where $0<y<u(\alpha)-b$ and $0<z<v(\alpha)-b$. Let $\bar{u}(\alpha)=y+b$ and $\bar{v}(\alpha)=z+b$. We note that if $u(\alpha)>0$ or $v(\alpha)>0$ then $\bar{u}(\alpha)$ and $\bar{v}(\alpha)$ may be chosen so that $0<\bar{u}(\alpha)<u(\alpha), 0<\bar{v}(\alpha)<v(\alpha)$, (but not necessarily both).

(13) If $\alpha \in K^{\prime}$ and

(i) $\bar{u}(\beta)=0$ for all $\beta \in K$ such that $\beta>\alpha$, let $\bar{u}(\alpha)=0, \bar{v}(\alpha)=x(\alpha)$,

(ii) otherwise, let $\bar{u}(\alpha)=x(\alpha), \bar{v}(\alpha)=0$.

Now let $U=\{\beta \in M(u) \mid$ for all maximal chains $C \subseteq \Gamma$ such that $\beta \in C$, either $C \cap M(x)=\varnothing$ or $C \cap M(w)=\varnothing\}$ and let $W$ be similarly defined for $M(v)$. Let $\Lambda=\{(\gamma, \delta) \in M(x) \times M(w) \mid \gamma \nmid \delta$ and there is $\lambda \in L$ such that $\lambda<(\gamma, \delta)\}$. Finally, if $U$ and $W$ are not empty, let $\Lambda(u)=U \times\{\min \{\gamma, \delta\} \mid(\gamma, \delta) \in \Lambda$ and $\max \{\gamma, \delta\} \in M(v)\}$ and $\Lambda(v)=W \times\{\min \{\gamma, \delta\} \mid(\gamma, \delta) \in \Lambda$ and $\max \{\gamma, \delta\} \in M(u)\}$. It follows that both $\Lambda(u)$ and $\Lambda(v)$ are totally disjoint.

The set $L \cap[S(x) \cup S(u) \cup S(v)]$, if not empty, contains a subset $N$ of all the maximal elements. Let $D$ be the trivial components of $N$,

$$
D(u)=\{\lambda \in D \mid \lambda<(\gamma, \delta) \in \Lambda(u)\}
$$

and

$$
D(v)=\{\lambda \in D \backslash D(u) \mid \lambda<(\gamma, \delta) \in \Lambda(v)\}
$$

By hypothesis there are disjoint subsets

$$
E_{1} \subseteq\{\lambda \in \Gamma \mid \lambda<(\gamma, \delta) \in \Lambda(u)\}
$$

and

$$
E_{2} \subseteq\{\lambda \in \Gamma \mid \lambda<(\gamma, \delta) \in \Lambda(v)\}
$$

such that $E_{1}$ covers $D(u)$ and $E_{2}$ covers $D(v)$. We again assume, for each $\beta \in E_{1} \cup E_{2}$ there is an element $\lambda \in D(u) \cup D(v)$ such that $\lambda<\beta$. Thus, $E_{1} \cup E_{2} \subseteq L$. Denote by $E^{\prime}$ the maximal elements of $E_{1} \cup E_{2} \cup(N \backslash D)$. If $D(u)$ or $D(v)$ is empty, we take $E_{1}$ or $E_{2}$ to be empty.

(14) If $\alpha \in L \mid\left(E^{\prime} \cup D\right)^{*}$, let $\bar{u}(\alpha)=\bar{v}(\alpha)=0$. 
(15) (i) If $\alpha \in E^{\prime}$ and $\alpha<\beta \in M(u)$ implies $C \cap M(w)=\varnothing$ for all maximal chains $C \subseteq \Gamma$ such that $\beta \in C$, let $0 \neq \bar{u}(\alpha)<u(\alpha), \bar{v}(\alpha)=x(\alpha)-\bar{u}(\alpha)$,

(ii) dual of (i).

(16) (i) If $\alpha \in E^{\prime}$ and $\alpha<\beta \in M(u)$ implies $C \cap M(x)=\varnothing$ for all maximal chains $C \subseteq \Gamma$ such that $\beta \in C$, let $0<\bar{u}(\alpha), \bar{v}(\alpha)=x(\alpha)-\bar{u}(\alpha)$,

(ii) dual of (i).

(17) Otherwise, for $\alpha \in L$ let $\bar{u}(\alpha)=x(\alpha), \bar{v}(\alpha)=0$.

A straightforward check shows that cases (1)-(17) are mutually exclusive and for any $\alpha \in \Gamma, \bar{u}(\alpha)$ and $\bar{v}(\alpha)$ are determined by one of these cases. Thus, $\bar{u}$ and $\bar{v}$ so defined are elements of the large direct sum of the $R_{y}$. Moreover, $S(\bar{u})$ and $S(\bar{v})$ are subsets of $S(u) \cup S(v) \cup S(x) \cup B^{\prime} \cup E^{\prime}$ where each set in this union satisfies the maximum condition. Thus, $\bar{u}, \bar{v} \in V$. Clearly, $\bar{u}+\bar{v}=x$.

If $\alpha \in M(\bar{u})$, then $\bar{u}(\alpha)$ can only be defined by (2), (3ii), (6ii), (7ii), (10i), (10ii), (12) or (16i). In all of these cases $\bar{u}(\alpha)>0$. Thus $\bar{u}>0$. If $\alpha \in M(\bar{v})$, then $\bar{v}(\alpha)$ can only be defined by (2), (3i), (6i), (7i), (10iii), (12) or (16ii). In all these cases $\bar{v}(\alpha)>0$. Thus $\bar{v}>0$.

Finally, if $\alpha \in M(v-\bar{v})$, then $\bar{v}(\alpha)$ can only be defined by (1), (3i), (5ii), (6ii), (7ii), (10ii), (11i), (12) or (15ii). In all these cases $v(\alpha)>\bar{v}(\alpha)$. If $\alpha \in M(u-\bar{u})$ then $\bar{u}(a)$ can only be defined by (1), (3ii), (5i), (6i), (7i), (9), (11ii), (12), or (15i). In all these $u(\alpha)>\bar{u}(\alpha)$.

Thus, we have constructed elements $\bar{u}, \bar{v} \in V$ such that $x=\bar{u}+\bar{v}$ and $0<\bar{u}<u$, $0<\bar{v}<v$. Hence, $V$ is a Riesz group.

IV. Proof of lemma-necessity. Let $V=V\left(\Gamma, R_{y}\right)$ be a Riesz group and let $\Delta$ be a totally disjoint subset of $\Gamma \times \Gamma$. Suppose $\Delta^{\prime}=\{\alpha \in \Gamma \mid \alpha<(\gamma, \delta) \in \Delta\}$ and let $A$ be a disjoint subset of trivial components of $\Delta^{\prime}$. For each $\gamma \in \pi_{1} \Delta \cup \pi_{2} \Delta$ choose $\alpha(\gamma) \in R_{\gamma}$ such that $\alpha(\gamma)>0$ and for each $\beta \in A$ choose $b(\beta) \in R_{\beta}$ such that $b(\beta) \neq 0$. Let

$$
\begin{array}{clll}
x(\alpha)=a(\alpha) & \text { if } \alpha \in \pi_{1} \Delta, & u(\alpha)=a(\alpha) & \text { if } \alpha \in \pi_{1} \Delta \\
=b(\alpha) & \text { if } \alpha \in A, & =0 & \text { otherwise; } \\
=0 & \text { otherwise; } & & \\
& & & \\
& v(\alpha)=a(\alpha) & \text { if } \alpha \in \pi_{2} \Delta, \\
& =0 & & \text { otherwise. }
\end{array}
$$

Then, $x, u$, and $v$ are strictly positive elements of $V$ such that $x \| u$ and $x \| v$. Moreover,

$$
\begin{aligned}
u(\alpha)+v(\alpha)-x(\alpha) & =0 & & \text { if } \alpha \in \pi_{1} \Delta \\
& =a(\alpha) & & \text { if } \alpha \in \pi_{2} \Delta \\
& =-b(\alpha) & & \text { if } \alpha \in A \\
& =0 & & \text { otherwise }
\end{aligned}
$$

so $0<x<u+v$. Thus, there are elements $\bar{u}, \bar{v} \in V$ such that $x=\bar{u}+\bar{v}$ and $0 \leqq \bar{u} \leqq u$, 
$0 \leqq \bar{v} \leqq v$. It easily follows that $M(\bar{u})=\pi_{1} \Delta, \bar{u}(\alpha)=u(\alpha)$ for all $\alpha \in \pi_{1} \Delta$, and for each $\beta \in M(\bar{v})$ there is an element $\delta \in \pi_{2} \Delta$ such that $\beta<\delta$.

If $\alpha \in A$, then $\bar{u}(\alpha) \neq 0$ or $\bar{v}(\alpha) \neq 0$ since $0 \neq x(\alpha)=\bar{u}(\alpha)+\bar{v}(\alpha)$. If $\bar{u}(\alpha) \neq 0$, then, since $\bar{u} \geqq 0$ and $u \geqq \bar{u}$, there is an element $\beta \in \Gamma$ such that $\beta>\alpha$ and $u(\beta)>\bar{u}(\beta)$. Now $\beta \notin \pi_{1} \Delta$ so $u(\beta)=0$ which implies $\bar{u}(\beta) \neq 0$. Thus, there is $\gamma \in \pi_{1} \Delta$ such that $\beta<\gamma$. Also, $\beta \notin A$, since $u(\beta)>\bar{u}(\beta)$ implies $\beta$ is ordered. Thus, $x(\beta)=0$ and $\bar{v}(\beta)=-\bar{u}(\beta) \neq 0$. Consequently, $\beta<\delta \in \pi_{2} \Delta$. This shows that, for each $\alpha \in A$, there is an ordered component $\beta$ such that $\alpha<\beta<(\gamma, \delta) \in \pi_{1} \Delta \times \pi_{2} \Delta$ and $\bar{u}(\beta) \neq 0 \neq \bar{v}(\beta)$.

Let $B=\left\{\beta \in \Gamma \mid \beta\right.$ is ordered $\beta>\alpha \in A, \beta<(\gamma, \delta) \in \pi_{1} \Delta \times \pi_{2} \Delta$ and $\left.\bar{u}(\beta) \neq 0 \neq \bar{v}(\beta)\right\}$. Then $B \subseteq S(\bar{u})$. If $B^{\prime}$ denotes the collection of maximal elements of $B$, then $B^{\prime}$ is a disjoint collection of ordered components that covers $A$. This completes the proof of the lemma.

V. Proof of the theorem. In this section we consider the case where an o-group $R_{y}$ need not be dense. If $R_{y}$ is discrete then there are elements $x, y \in R_{y}$ such that $x<y$, and $x \leqq z, z \leqq y$ implies $z=x$ or $z=y$. If $0 \leqq a \leqq y-x$ then $x \leqq x+a \leqq y$, so $a=0$ or $a=y-x$. Thus, every discrete $R_{\gamma}$ contains a minimum strictly positive element. We will denote this element by $m(\gamma)$. We now prove the main theorem.

THEOREM. Let $\Gamma$ be a po-set and for each $\gamma \in \Gamma$, let $R_{\gamma}$ be a trivially ordered group or an o-group. Then $V=V\left(\Gamma, R_{\gamma}\right)$ is a Riesz group if and only if every totally disjoint subset of $\Gamma \times \Gamma$ and every disjoint set of discrete components of $\Gamma$ is complete.

Proof. The only place, in the proof of the sufficiency of the lemma, where density is used is in (12) and (13). We confine our attention to these and assume everything else is defined as before. Using the same notations as before, let

$$
K=\left\{\lambda \in \Gamma_{3} \mid \lambda \in M(x) \cup M(u) \cup M(v) \cup M(w)\right\}
$$

and let

$$
K^{\prime}=\left\{\sigma \in \Gamma_{3} \mid \sigma<\lambda \text { for some } \lambda \in K\right\}
$$

Let $Y=\{\lambda \in K \cap M(w) \mid \lambda$ is discrete $\}$. If $\alpha \in K \cap M(w)$ but $\alpha \notin Y$, then $\alpha$ is dense so define $\bar{u}(\alpha)$ and $\bar{v}(\alpha)$ by (12). Thus, let $\alpha \in Y$.

(12a) If $\alpha \notin M(u)$, let $\bar{u}(\alpha)=x(\alpha)-v(\alpha), \bar{v}(\alpha)=v(\alpha)$.

(12b) If $\alpha \in M(u)$ but $\alpha \notin M(v)$, let $\bar{u}(\alpha)=u(\alpha), \bar{v}(\alpha)=x(\alpha)-\bar{u}(\alpha)$.

If $\alpha \in M(u) \cap M(v)$ and

(12c) $x(\alpha)<u(\alpha)$, let $\bar{u}(\alpha)=x(\alpha), \bar{v}(\alpha)=0$,

(12d) $x(\alpha)>u(\alpha)$, let $\bar{u}(\alpha)=u(\alpha), \bar{v}(\alpha)=x(\alpha)-\bar{u}(\alpha)$,

(12e) $x(\alpha)=u(\alpha)$ and $x(\alpha)<v(\alpha)$, let $\bar{u}(\alpha)=0, \bar{v}(\alpha)=x(\alpha)$,

(12f) $x(\alpha)=u(\alpha)$ and $x(\alpha)>v(\alpha)$, let $\bar{v}(\alpha)=v(\alpha), \bar{u}(\alpha)=x(\alpha)-\bar{v}(\alpha)$,

(12g) $x(\alpha)=u(\alpha)=v(\alpha)$, let $\bar{u}(\alpha)=m(\alpha), \bar{v}(\alpha)=x(\alpha)-m(\alpha)$.

Let $T=\{\lambda \in Y \mid u(\lambda)=v(\lambda)=x(\lambda)=m(\lambda)\}$ and $T^{\prime}=\left\{\sigma \in \Gamma_{3} \mid \sigma<\lambda\right.$ for some $\left.\lambda \in T\right\}$. If $D$ is the collection of maximal components of $T^{\prime} \cap[S(x) \cup S(u) \cup S(v)]$ and $A$ is the set of trivial components of $D$, then $A$ is a disjoint subset of $\{\lambda \in \Gamma \mid \lambda<\sigma$ for 
some $\sigma \in T\}$. Since $T \subseteq M(w), T$ is a disjoint set of discrete components. Thus, by hypothesis, there is a disjoint subset $B \subseteq\{\lambda \in \Gamma \mid \lambda<\sigma$ for some $\sigma \in T\}$ such that $B$ contains no trivial components and $B$ covers $A$. We again assume for each $\beta \in B$ there is $\alpha \in A$ such that $\beta>\alpha$. Thus, $B \subseteq \Gamma_{3}$.

Let $B^{\prime}$ denote the maximal components of $B \cup(D \backslash A)$.

(13a) If $\alpha \in T^{\prime} \backslash\left(B^{\prime}\right)^{*}$, let $\bar{u}(\alpha)=0, \bar{v}(\alpha)=0$.

(13b) If $\alpha \in T^{\prime}$ and $\alpha<\beta$ for some $\beta \in B^{\prime}$, let $\bar{u}(\alpha)=x(\alpha), \bar{v}(\alpha)=0$.

(13c) If $\alpha \in B^{\prime}$ let $\bar{v}(\alpha)>\{0, x(\alpha)-u(\alpha)\}, \bar{u}(\alpha)=x(\alpha)-\bar{v}(\alpha)$.

(13d) If $\alpha \in K^{\prime} \backslash T^{\prime}$ and there is $\beta \in Y$ such that $\beta>\alpha$ and $\bar{u}(\beta)$ is defined by (12b) or (12d), let $\bar{u}(\alpha)=u(\alpha), \bar{v}(\alpha)=x(\alpha)-\bar{u}(\alpha)$.

(13e) If $\alpha \in K^{\prime} \backslash T^{\prime}$ and no $\beta \in Y$ exists such that $\beta>\alpha$ and $u(\beta)$ is defined by (12b) or (12d), but there is $\gamma \in Y$ such that $\gamma>\alpha$ and $\bar{u}(\gamma)$ is defined by (12a) or (12f), let $\bar{v}(\alpha)=v(\alpha), \bar{u}(\alpha)=x(\alpha)-\bar{v}(\alpha)$.

(13f) If $\alpha \in K^{\prime} \backslash T^{\prime}$ and for all $\beta \in K$ such that $\beta>\alpha, \bar{u}(\beta)=0$ but $\bar{u}(\beta)$ is not defined by (12a), let $\bar{u}(\alpha)=0, \bar{v}(\alpha)=x(\alpha)$.

(13g) Otherwise, for $\alpha \in K^{\prime} \backslash T^{\prime}$, let $\bar{u}(\alpha)=x(\alpha), \bar{v}(\alpha)=0$.

It follows that the above cases are mutually exclusive and exhaust $Y \cup K^{\prime}$. Moreover, $\bar{u}$ and $\bar{v}$ are defined so that $\bar{u}, \bar{v} \in V$ and $x=\bar{u}+\bar{v}$. The maximal components of $\bar{u}$ can occur only at (12b), (c), (d), (f) and (g), and those of $\bar{v}$ can occur only at (12a), (d), (e), (f), (g) and (13c) and in all cases are strictly positive. The maximal components of $u-\bar{u}$ can occur only at (12a), (c), (e), (f), (g) and (13c), and those of $v-\bar{v}$ can occur only at (12b), (c), (d), (e), and (g) and in all cases are strictly positive. Therefore $0<\bar{u}<u$ and $0<\bar{v}<v$. Thus, $V$ is a Riesz group.

Suppose now that $V$ is a Riesz group. Since the proof of necessity in the previous lemma does not depend on the density of the o-group, it only remains to show every disjoint set of discrete components is complete. To this end let $T$ be a disjoint set of discrete components and $A$ a disjoint subset of trivial components of $\{\lambda \in \Gamma \mid \lambda<\sigma$ for some $\sigma \in T\}$.

Well order $T$, and for each $\beta \in T$ let $A(\beta)=\{\alpha \in A \mid \alpha<\beta$ and $\alpha \nless \eta$ for all $\eta \in T$ where $\eta<\beta$ in the ordering of $T\}$. If $A(\beta) \neq \varnothing$, define $x, u$ and $v$ as

$$
\begin{aligned}
& x(\alpha)=m(\alpha) \quad \text { if } \alpha=\beta, \quad u(\alpha)=v(\alpha)=m(\alpha) \quad \text { if } \alpha=\beta, \\
& =b(\alpha) \neq 0 \text { if } \alpha \in A(\beta), \quad=0 \quad \text { otherwise. } \\
& =0 \quad \text { otherwise; }
\end{aligned}
$$

Then, $x, u, v$ are positive elements of $V, x\|u, x\| v$ and $x<u+v$. Thus, elements $\bar{u}$ and $\bar{v}$ exist such that $x=\bar{u}+\bar{v}$ and $0 \leqq \bar{u} \leqq u, 0 \leqq \bar{v} \leqq v$.

Either $\bar{u}(\beta)=m(\beta)$ or $\bar{v}(\beta)=m(\beta)$. Suppose $\bar{u}(\beta)=m(\beta)$, then $\bar{v}(\beta)=0$. If $\alpha \in A(\beta)$ and $\bar{u}(\alpha) \neq 0$ then $u \geqq \bar{u}$ implies there is a $\gamma$ such that $\beta>\gamma>\alpha$ and $\bar{u}(\gamma)<0$. But $x(\gamma)=0$ so $\bar{v}(\gamma)=-\bar{u}(\gamma)>0$. If $\bar{u}(\alpha)=0$, then $\bar{v}(\alpha) \neq 0$ and $\bar{v} \geqq 0$ implies there is a $\delta$ such that $\beta>\delta>\alpha$ and $\bar{v}(\delta)>0$. Again $x(\delta)=0$ so $\bar{u}(\delta)=-\bar{v}(\delta)<0$. Thus, for each $\alpha \in A(\beta)$ there is a nontrivial component $\gamma$ such that $\alpha<\gamma<\beta$ and $\bar{u}(\gamma) \neq 0 \neq \bar{v}(\gamma)$. Let $B(\beta)=\{\gamma \in \Gamma \mid \gamma$ is nontrivial, $\gamma<\beta, \bar{u}(\gamma) \neq 0 \neq \bar{v}(\gamma)$ and $\gamma>\alpha$ for some $\alpha \in A(\beta)\}$. 
Then $B(\beta) \subseteq S(\bar{u})$. Let $B^{\prime}(\beta)$ be the maximal components of $B(\beta)$. Then $B^{\prime}(\beta)$ is a disjoint set that covers $A(\beta)$. If $A(\beta)=\varnothing$ let $B^{\prime}(\beta)=\varnothing$.

Now if $\beta, \eta \in T$, then $B^{\prime}(\beta) \cup B^{\prime}(\eta)$ is a disjoint set. To show this we need only consider $\lambda \in B^{\prime}(\beta), \sigma \in B^{\prime}(\eta)$ and $\lambda<\sigma$. There exist elements $\alpha_{1} \in A(\beta)$ and $\alpha_{2} \in A(\eta)$ such that $\alpha_{1}<\lambda$ and $\alpha_{2}<\eta$. If $\beta<\eta$ in the ordering of $T$, then $\alpha_{2} \notin A(\eta)$ and if $\eta<\beta$, then $\alpha_{1} \notin A(\beta)$. Thus, $\bigcup_{\beta \in T} B^{\prime}(\beta)$ is a disjoint subset of $\{\lambda \in \Gamma \mid \lambda<\sigma \in T\}$. Moreover, $\bigcup_{\beta \in T} B^{\prime}(\beta)$ contains no trivial components and covers $A$. This completes the proof of the theorem.

\section{REFERENCES}

1. P. Conrad, J. Harvey and C. Holland, The Hahn embedding theorem for abelian lattice ordered groups, Trans. Amer. Math. Soc. 108 (1963), 143-169.

2. J. R. Teller, On partially ordered groups satisfying the Riesz interpolation property, Proc. Amer. Math. Soc. 16 (1965), 1392-1400.

GeORGETOWN UNIVERSITY,

WaSHINGTON, D. C. 